\title{
Initial Observations on Economics, Pricing, and Penetration of the Internet of Things Market
}

\author{
Jens-Matthias Bohli, Christoph Sorge, and Dirk Westhoff \\ NEC Laboratories Europe \\ Kurfürsten-Anlage 36 \\ 69115 Heidelberg, Germany \\ \{bohli,sorge,westhoff $\} @$ nw.neclab.eu
}

This article is an editorial note submitted to CCR. It has NOT been peer reviewed. The author takes full responsibility for this article's technical content. Comments can be posted through CCR Online.

\begin{abstract}
One expectation about the future Internet is the participation of billions of sensor nodes, integrating the physical with the digital world. This Internet of Things can offer new and enhanced services and applications based on knowledge about the environment and the entities within. Millions of micro-providers could come into existence, forming a highly fragmented market place with new business opportunities to offer commercial services. In the related field of Internet and Telecommunication services, the design of markets and pricing schemes has been a vital research area in itself. We discuss how these findings can be transferred to the Internet of Things. Both the appropriate market structure and corresponding pricing schemes need to be well understood to enable a commercial success of sensor-based services. We show some steps that an evolutionary establishment of this market might have to take.
\end{abstract}

\section{Categories and Subject Descriptors}

K.6.0 [Management of Computing and Information Systems]: General-Economics

\section{General Terms}

Economics

\section{Keywords}

Sensor networks, Internet of Things, Pricing, Market

\section{INTRODUCTION}

Wireless sensor network (WSN) islands for the civilian sector have reached a stage in which they can actually be deployed outside research labs and provide useful information (see, e.g., [6]). Current sensor networks are typically used by their operators for their own purposes only, but a change is envisioned: End-users shall be enabled to request environmental data from various hot-spots all over the world. This is based on the vision that millions of micro-providers will deploy and manage sensor network islands locally and, in a next step, open them to the public. A trading service in the Internet - operated by a search engine provider or a service provider purely dedicated to the composition and trading of sensor services - will then ensure that requests for sensor information will be sent to the right WSN island. This trading service can also take care of payments, thus forming the basis for a new market. We will refer to this envisioned market as the Internet of Things (IoT) Market. A research project actually considering such a vision is the European project SENSEI ${ }^{1}$.

While technical challenges are currently being addressed in research, the economic and business aspects of establishing the IoT market leave some more uncertainties:

1. What could IoT services look like?

The primary service is certainly the provision of sensor data. A higher level service can aggregate data from several sources to produce more accurate data, or process data as context information. However, collateral services could also be imagined, e.g. the WSN could be rented as a whole to run an end-user's own software on it.

2. What can be the incentives to provide information? Organizations and also private micro providers that participate in the system need incentives to offer services and access to their WSNs. It is, however, unclear if a customer will ever be willing to pay "micro services", such as single sensor network queries. It might be more realistic to assume that profit will be made by personalized advertisement that is enabled by the sensor information.

3. How to initiate the system?

A major question is under what circumstances a 'critical mass' of WSN owners is willing to deploy their networks and open it to the public. As long as no customers are available it is certainly not worth to deploy a WSN, while based on sparsely deployed WSNs, no attractive services will be possible.

Obviously, finding appropriate answers to the above questions highly depends on the actual phase we consider this market to be in. For example, once the system has been accepted and deployed on a large scale, the incentives for participating in the market will no longer be an issue. From our point of view, the most challenging phase starts once

\footnotetext{
${ }^{1}$ http://www.ict-sensei.org/
} 
most technical problems have been solved, but the system is only used by technical experts. How can additional players, wishing to make profit, be attracted?

The following sections will try to find answers to these questions. We start by identifying the players in the IoT market (section 2). Next, we discuss strategies of these players, in particular concerning the pricing of services, in section 3. While these considerations assume an existing market, specifics of the bootstrapping phase are discussed in section 4 . We conclude this paper in section 5 .

\section{ENTITIES IN THE IOT MARKET}

This section describes the entities we anticipate to play a role in a future WSN market. These are i) providers of sensor information or services that base on sensor information, ii) consumers querying information or iii) intermediaries who provide the market platform to connect providers and customers.

\subsection{Providers}

We can think of two categories of service providers: Those providing raw sensor information and those building higher level services.

\subsubsection{Sensor Information}

Sensors and sensor networks are the source of information. Some of them include just a single or a few sensors, others will probably be based on larger-scale wireless sensor networks. Sensor information is already widely used for many purposes. Sensors and sensor networks that are deployed or planned come in different sizes and shapes. For example, hobby meteorologists measure temperature and humidity in their garden; vehicles sense information about road condition, their own location, and velocity; roadside sensor networks can provide information about road conditions to increase traffic safety. On a large scale, (mostly wired) sensor networks are already used to monitor critical infrastructures or industrial processes in SCADA systems. While these will probably remain closed systems, other networks may be open for the public - e.g. city administrations gathering data about free parking spots, water and shipping authorities monitoring water levels, or other environmental monitoring systems.

The vision of the Internet of Things assumes that a large number of objects that will be produced in the future will include a networking device. There can be cheap objects which only announce their presence as well as high-class objects which will also provide information about their status and environment. As described above, various sensor systems are already deployed even today. It can be expected that the number of sensor operators will grow further. The sensor systems are deployed by individuals or organizations for their own purposes. Indeed, without a specific need for this information, it might not be worth to invest in collecting this information. However, once raw information is produced by those sensor systems, it can well be supplied in a global sensor information system. In an IoT market, those sensor network operators would offer their information in a world-wide system for customers interested in the information or businesses offering their customers services that can be improved by access to the sensor information. Potential privacy implications can be resolved by pre-processing the data or providing them with an according policy. This mo- tivates the importance of micro providers. We summarize this in

Thesis 1: On the supply side of the IoT
market, micro providers will play an impor-
tant role.

\subsubsection{Composed Services}

Another aspect of the IoT market is the availability of higher level (composed) services than the raw information made available by the micro providers. This raw information, being provided by a specific sensor or sensor network, can be the temperature at a defined spot, the traffic density on a road segment, or the workload on a specific machine. High-level information services can be offered by aggregating this kind of data. Information from separate sensors and sensor networks in different domains can be evaluated to get information on a larger scale or about events that need several sensors' information to detect. Besides the pure aggregation of information, many kinds of services can be enabled or enhanced by sensor information. One can, for example, think of a calendar of events giving recommendations for spare-time activities based on information on the weather or congestion.

Thesis 2: Sensor information should be
made available on an open platform to allow
everyone to offer higher level services.

It should be possible for everyone to collect necessary data and process them to offer a new service. By this, creativity and effort of many participants can be used to generate a higher value of the system. Similarly to micro providers on the sensor level, micro providers for composed services will appear.

Of course, data providers themselves are free to build their own services on top of the information they provide - and some of them already do. Water and shipping authorities, for example, publish water levels on web pages and derive flood forecasts. Still, making the original sensor readings available for processing by others makes sense, as it allows third parties to compose services.

\subsection{Consumers}

Consumers request information or services, which they use for their own advantage. The above-mentioned service providers are providers and consumers at the same time: They consume lower level services and raw information to provide their customers higher level services.

Consumers wanting to use information services will probably be interested in more than a single sensor reading. In general terms, information services have a higher utility for their users

- the more information sources there are available

- the more high-level services are composed of the single information services.

We will deal with other aspects influencing the consumers' valuation in the following sections.

\subsection{Intermediaries and service providers}

Understanding potential market structures of future WSN markets may be eased when having a look at the transaction 
cost theory of the firm. This theory has been suggested by Coase [4] in 1937, but is still used today to explain certain phenomena in business management (see, e.g., [1]). Basically, the theory explains why firms exist instead of coordinating all actions in an economy using the price mechanism. Consider, for example, engineers designing a car. In theory, they could sell each development separately instead of having a permanent contract. The reason for not using the price mechanism both in this example and in general is that transaction costs would be too high. For example, the mentioned engineers would have to regularly negotiate new contracts, and the car manufacturers would invest more time in the search for new ideas. Coordinating the production of any complex good (like a car) would become prohibitively expensive without the existence of firms. As a result, we can state that, especially for large economic systems, the price mechanism alone does not suffice; instead, coordination has to be achieved by introducing firms or intermediaries.

Transaction cost theory also applies to the provision of WSN services: If consumers interact with a large number of WSN island providers or service providers, they will not be willing to conclude hundreds of individual contractsmoreover, the transaction costs caused would probably become too high. Therefore,

\section{Thesis 3: Intermediaries are needed as co- ordinating central structures on the IoT mar- ket.}

These intermediaries make it possible for consumers and for providers to have only one business partner each. Intermediaries can take care of authentication, authorization, accounting and billing, and the infrastructure needed to find appropriate services.

In addition, most users will find a system more useful if they do not just receive individual sensor readings. Composed services, as discussed in section 2.1.2, can add significant value. So, besides the WSN island providers, there is also a need for providers of composed services. These might be identical to the above-mentioned intermediaries, but in principle, this is not necessary.

\section{PRICING SCHEMES AND STRATEGIC BEHAVIOR}

Finding a pricing scheme that is suitable for consumers, micro providers and intermediaries is a challenging task. We have a look at existing insights from information economy first and then try to apply them to pricing for WSN-based services.

\subsection{Pricing schemes in the information econ- omy}

Pricing of information goods is different from traditional pricing, e.g. for physical goods: While fixed costs for information goods tend to be high, the marginal costs for producing additional units of the same good are typically close to zero. Therefore, cost-based pricing does not help. However, some instruments have been described by economists as being particularly suitable for information goods (for a good overview, see [9]). Price discrimination describes the attempt to charge different prices, depending on the customers' willingness to pay. Since customers do not tend to reveal the fact that they are willing to pay a high price, tricks like versioning are applied: The same basic good is sold in different versions, e.g. highly accurate sensor readings and (intentionally) fuzzy measurements. While the costs may be the same (or, in some cases, even higher for the lower quality version), the high quality version can be sold at a high price, while the low quality version can generate additional revenues from customers with a low willingness to pay. In the IoT market, the freshness of information will often be important, so the low quality version might just be a bit older (the same principle has been applied in the context of stock quotes before [9]). Another mechanism is bundling, i.e. selling two ore more goods as a set (see [10] for guidelines on bundling applicable to the IoT market). It can be shown that, under reasonable assumptions, bundling information goods allows the seller to acquire a larger part of the consumer's willingness to pay than selling single products [2].

\subsection{Pricing for WSN-based services}

Bundling is also the first choice when selling sensor information to consumers. For example, it makes more sense to sell access to all sensors in a city than just to an individual one. Aside from the theory mentioned above, charging individual queries would also require a micro payment scheme. Moreover, flat pricing has proven to be popular and beneficial, e.g. for telephony services and internet access [7].

Micro providers, on the other hand, should not receive a flat payment which is independent of the number of queries they answer. If they would, there might not be an incentive for them to offer useful services. Note that bootstrapping remains a problem: As long as the customer base is small, there are no signals to indicate which WSN services are most useful. Micro providers have to rely on their own expectations at this point in time, trying to increase their future revenues.

Since the requirements are very different, we introduce

Thesis 4: The pricing schemes for con-
sumers and for micro providers should be de-
coupled.

This means that the scheme by which a micro provider is paid need not be identical to the pricing scheme used for the consumer using its services. Obviously, this is only possible if intermediaries are introduced. There is no need to have just one pricing scheme each for consumers and micro providers. Instead, we suggest

Thesis 5: Intermediaries can offer schemes
tailored to the preferences of certain con-
sumer groups, and a competition of pricing
schemes can evolve among intermediaries.

Both versioning and bundling, as discussed above, are likely to be part of these pricing schemes.

\subsection{Co-opetition: Intermediaries}

It is not enough for intermediaries to develop a pricing strategy to maximize profits. There are likely to be more players in the market, so competition will arise. If more than one intermediary exists, each of them will have contracts with some sensor network providers. However, its customers may also want to get access to sensor data provided by other providers, which in turn have contracts with 
other intermediaries. High-level composed services might also require simultaneous access to a large number of sensor network islands. So, just like in case of telecommunication providers, the consumers' utility can be increased by cooperation of the intermediaries. This way, customers of one intermediary can access sensor networks associated to other intermediaries. On the other hand, intermediaries are still competitors, so they will also try to differentiate their services. A similar situation occurs in case of fixed and mobile telephony providers. Economists refer to this as "co-opetition"; the topic has attracted some attention in economics [3], [5].

In summary, we propose

Thesis 6: Intermediaries have to cooperate to maximize the customer's utility (and, eventually, their own profits), while also trying to differentiate their services.

\section{GETTING THERE: PENETRATION}

Let us for the moment assume that the pervasive availability of WSNs and WSN-based services would be very useful, and that people would also be willing to pay for them. Still there is the classical chicken-and-egg problem - whether this can be solved will decide if an IoT market comes into existence at all: Currently, there are only few WSN islands available; where the sensor networks exist, they offer private services for closed user groups, which makes it difficult to compose useful services from different islands. But as long as there are no useful services, customers will not be willing to pay, which means there is little incentive for anyone to deploy additional WSN islands and to make them available to the public.

We therefore try to provide some insights concerning the bootstrapping of the IoT market.

\subsection{Market penetration}

The diffusion of innovations has been described by Rogers [8] with an S-curve (logistical curve), with a small number of early adopters being the first to adopt a new technology. Only after the innovation has proven useful to them, it gets adopted by a large number of users. Once a high market penetration has been reached, the number of additional units sold goes down again. Besides this classical diffusion theory, network effects often play a prominent role in the information economy. Network effects occur when the utility of a good for a customer depends on the number of users of that product. For example, a fax machine is rather useless if you are the only person in the world that has one; but if there are millions of users, its utility rises, as documents can be easily exchanged with all these users (see, e.g., [9]).

A significant investment is needed to introduce an innovative product. Firstly, early adopters need to be convinced of the product's potential usefulness. Secondly, the product's utility is still very limited at this point in time if it depends on the number of adopters. Therefore, it may even make sense to give the product away for free until there are enough adopters to make it useful (while having a strategy in mind to introduce payments later, e.g. for additional services while sustaining a free base service). To make matters worse, generating useful IoT products depends on the cooperation of many parties, and the market to be penetrated does not even exist yet. In a way, the market itself is the first product that needs to be adopted.

\subsubsection{The cost perspective}

Two observations, however, indicate that an IoT market may have a more smooth penetration phase than other IT markets. Both contribute to low initial costs for the market. Firstly, the initial costs $C$ of a world-wide IoT network are relatively low: large parts of the communication infrastructure are already available. Only the roll-out of the last meters for the WSN islands and their gateways is missing. In comparison, the costs of higher level service providers and intermediaries can be neglected, since they build on an existing infrastructure and do not need significant hardware investment. Secondly, these fixed costs are distributed over all micro-providers who intend to join the IoT service market. In other words,

Thesis 7: the total cost of the IoT network
is the sum of the costs $c_{i}$ for the WSN is-
lands to be connected: $C=\sum c_{i}$. The initial
costs $c_{i}$ are borne by each micro-provider on
its own.

Both the distribution of the costs over all micro-providers and the existence of an infrastructure may very much help reaching a critical mass in the penetration phase. On the other hand, from the perspective of the micro-provider, the IoT service market itself is likely to be a side issue. The motivation for deploying a WSN service lies elsewhere. In other words: A WSN island micro-provider who enters the IoT end-user service market will most probably have established its core-business (private service) with the WSN island roll-out.

\subsubsection{The value perspective}

Considering the costs alone, no business would exist in the world. Therefore, we also have to look at the value created by the IoT market.

Network effects occur in a situation in which the utility that users get from a technology depends on the number of other people using the technology. Note that the utility of sensor information services does not directly depend on the number of users (as it is the case for pure communication services). However, a feedback loop exists: The more information services there are, the higher the utility for users becomes. As a consequence, more users will actually use information services, possibly creating additional incentives for micro providers to offer their services.

For telecommunication networks, Metcalfe's law (cmp. [9]) describes the overall network value depending on the number of its users (or terminal devices): If the value of the network for each user of the network is proportional to the number of users $n$, then the total value of this network is proportional to $n \cdot(n-1)=n^{2}-n$ (where the term $-n$ can be neglectedanyway, this is not a precise mathematical law). Given the presence of (indirect) network effects also in the case of the Internet of Things, we can expect similar results. To be more concrete, we assume

- the utility for the consumers and, consequently, the number $n$ of consumers to grow with the number of WSN islands, 
- the number of WSN islands to grow with the number $n$ of consumers.

While Metcalfe's law is based on the number of possible communication connections, the situation is more complex for the IoT market than in case of telecommunication networks: The Internet of Things is not mainly about communication between end users. Still, the positive feedback in both direction leads to

Thesis 8: The value of the IoT market
grows more than linearly with the number of
consumers.

The existence of network effects, supports our assumption that intermediaries will come into existence: once a critical mass of WSN island micro-providers opens their services to the public, they will want to participate in order to get their share of the total value of the IoT market.

\subsubsection{The role of intermediaries}

Intermediaries are the gluing element for the IoT market. It is therefore foreseeable that an intermediary's share of the whole IoT market value, as described in thesis 8 , will be by orders of magnitude larger than the one of a micro provider.

The above section contains good and bad news for the vision of a worldwide IoT market at the same time:

1. Intermediaries: An intermediary can join the market with almost no initial costs from his side and the reasonable expectation for highest revenue. Even the coexistence of others would not necessarily mean a reduction of revenue: increasing the size of the overall cake while reducing the shares of individuals might still result in larger pieces for all players.

2. Micro-providers: From a micro-provider's perspective, the expected profit (if any) is much lower: only for a sufficiently large number of consumers, the overall IoT market value (and, as a consequence, the micro provider's share) is sufficient to recover the costs.

So, the basic dilemma is that those who take the investment (WSN island micro-providers), at a first glance, only have moderate profit expectations. Intermediaries should be aware of this dilemma and may try to solve it:

Thesis 9: Intermediaries should consider
subsidizing micro providers to create an ad-
ditional incentive for service provisioning and
enable the intermediaries' business in the
first place.

\subsubsection{Protecting values}

Though profit expectations for micro providers are moderate, there is one point in favor of participation in the IoT markets. A fundamental rule of information technology does not hold anymore for IoT markets in its strict sense: In its general form, information delivered over a network in digital form shows the first-copy problem. Once the first copy of the digital information is produced, additional copies cost essentially nothing. The fact that information are costly to produce but cheap to reproduce (see also [9]) is not only true for the owner of the good but also useful for product piracy. Luckily, the digital information copy problem is fundamental different for the IoT market:
Thesis 10: For the IoT market, the copy
problem of digital information is moderated.

Freshness and timely deliver data queried from a WSN are, to a large extent, as important as the raw data themselves. Copying and distribution of the received data by a non-owner of a WSN island (end-user or intermediary) will not necessarily affect the micro provider's business. Since a WSN island's answer to a query can change any time, the value of the query for an end-user is always the same and does not reduce once having requested the service. This holds particularly for fast changing information such as location information or event detection. If the service offers access to historical data, the situation gets similar to ordering digital books or songs. Here, the same customer will most likely not order the same good again.

Thesis 10 may be the convincing argument for a critical mass of micro-providers to provide their WSN islands to the public. The joining of intermediaries will then no longer pose a problem.

\subsection{Bootstrapping the IoT market}

Some strategies and principles allowing the IoT market to take off can be deducted from the observations stated above. Take the consumers as a starting point. Initially, they will see a system with very limited value for them. One way to still make them buy the product and get a sufficient number of consumers is to start with very low prices:

Thesis 11: Initial prices must be low.

Services might even be offered for free in this phase. A free base service might be offered to all customers who are micro providers at the same time, giving an additional incentive to provide services.

Obviously, offering low prices is not enough: The value of the product also has to be increased, meaning that as many information services as possible should be made available. They are based on sensor readings, which we expect to be made available by micro providers (as stated above). These micro providers can be helpful for the penetration, as the task of deploying WSNs on a large scale will not be manageable by a single provider alone. They should not be prevented from entering the market by high costs.

\begin{tabular}{l} 
Thesis 12: Low entry costs: The cost of \\
deploying and integrating a sensor network \\
into the system must be low. \\
\hline
\end{tabular}

Of course, sensor hardware has to be paid for, and largescale WSNs will cost a significant amount of money even though electronic equipment usually becomes cheaper over time. But the WSNs may be needed for their operator's own purposes, anyway. They might, for example, be deployed for home/facility monitoring, or intelligent transport systems. In these cases, making their information available for the IoT market is not the primary purpose, but an additional value that can be provided. However, the additional capabilities for the IoT market must come at virtually zero costs. In addition, being forced to complement a service in order to make it more interesting for IoT consumers would also increase costs. We therefore introduce what might be called the Wikipedia principle: 


\section{Thesis 13: Provisioning of incomplete ser- vices should be allowed.}

Providing, say, the humidity of one's garden is not interesting per se, and no one would subscribe to the sensor information service just to get this information. However, it is a contribution and once a whole region provides this data, an information service on the distribution of rainfalls can be provided. Thus, we believe that even small incomplete contributions should be honored and rewarded by the system.

\subsubsection{Incentives for Providers}

We conjecture that

Thesis 14: Incentives will be needed to
stimulate participation of a large number of
(micro) providers.

Only in some cases, incentives might not be necessary. Examples include public authorities or non-profit organizations, like in the example of water and shipping authorities: They might provide information for the public good, as they are publicly financed. Also, individuals may see information provisioning as part of a hobby, and provide information without further incentives. However, this does not mean that additional incentives would not be helpful.

Several kinds of incentives are possible which could motivate providers to offer data or services in a framework. One option are providers that charge customers for their services.

An incentive for businesses could also be to provide information as a unique selling point to distinguish from their competitors. This means an extra service for their customers, and could therefore be suitable as a marketing measure.

\subsubsection{Finding services}

Let us get back to the consumers. Even if there is a sufficient number of services available, and these services come at a low cost - how should anyone know about them? Whether it is a local weather forecast or information about free parking spots: Based on the user's context and interests, there must be a way to let him know about the services he might find useful. This leads to

\section{Thesis 15: An efficient search mechanism is needed to find useful services.}

Existing search engine providers are probably in the best position to provide this search mechanism, which is a key requirement for the IoT market.

\section{CONCLUSION}

In this paper, we have described the current vision of a Internet-of-Things market, which offers a platform to collect and trade all kinds of sensor information. Besides the (micro) providers of sensor networks and the consumers interested in information services, we have identified the intermediaries as key players. We have identified and postulated in 15 theses what we believe to be the main characteristics of the IoT market, as well as guidelines for its participants. Keeping these in mind, there seems to be a reasonable chance for the envisioned market to come into existence and to create value for providers, consumers, and intermediaries.

The intent of this paper is not to solve all economic problems related to sensor information services, but rather to provide some ideas based on experience in other markets, and to stimulate discussion about the theses presented.

The authors admit that the decision to write this editorial note was mainly stimulated by their original doubt that something like the IoT market would ever be able to exist. However, when writing these initial thoughts on the economics, pricing and penetration of an IoT market, we tend to believe more in the power of such a market. As mandatory pre-conditions to survive its early stage, we highlight Thesis 8 and Thesis 10 as the two observations which stimulate the micro-providers and the intermediaries to join the market.

\section{ACKNOWLEDGMENT}

The authors would like to thank Mario Lischka for valuable comments.

\section{REFERENCES}

[1] B. A. Aubert, S. Rivarda, and M. Patry. A transaction cost model of it outsourcing. Information 8 Management, 41(7):921-932, 2004.

[2] Y. Bakos and E. Brynjolfsson. Bundling and competition on the internet. Marketing Science, 19(1):63-82, 2000.

[3] A. Brandenburger and B. Nalebuff. Co-Opetition: A Revolution Mindset That Combines Competition and Cooperation; The Game Theory Strategy That's Changing the Game of Business. Bantam Dell, 1996.

[4] R. H. Coase. The Nature of the Firm. Economica, 4(16):386-405, November 1937.

[5] D. R. Gnyawali, J. He, and R. Madhavan. Impact of co-opetition on firm competitive behavior: An empirical examination. Journal of Management, 32(4):507-530, 2006.

[6] S. Kim, S. Pakzad, D. Culler, J. Demmel, G. Fenves, S. Glaser, and M. Turon. Health monitoring of civil infrastructures using wireless sensor networks. Technical Report UCB/EECS-2006-121, University of California at Berkeley, 2006.

[7] A. M. Odlyzko. Internet pricing and the history of communications. Computer Networks, 36(5/6):493-517, 2001.

[8] E. M. Rogers. Diffusion of Innovations. Free Press, 5th edition, 2003.

[9] C. Shapiro and H. Varian. Information Rules. Harvard Business School Press, Boston, 1999.

[10] G. Xianjun, M. B. Stinchcombe, and A. B. Whinston. Bundling information goods of decreasing value. Management science, 51(4):662-667, 2005. 